\title{
ON TWO VARIABLE FUNCTIONAL INEQUALITY AND RELATED FUNCTIONAL EQUATION
}

\section{MirosŁAW ADAMEK}

Abstract. In this paper we show that solutions for a large class of functional inequalities can be obtained from solutions of the corresponding functional equations.

Mathematics subject classification (2000): Primary 26B25, 39B62.

Keywords and phrases: Functional equation and inequality, Gauss-composition, convexity.

\section{REFERENCES}

[1] M. Adamek, K. Nikodem, Zs. PÁles, On ( $K, \lambda)$-convex set-valued maps, Radovi Matematički, 11 (2003), 183-191.

[2] Z. DARÓCZY, Zs. PÁLES, Gauss-composition of means and the solution of the Matkowski-Sutô problem, Publ. Math. Debrecen, 61 (2002), 157-218.

[3] J. MatKows KI, M. WróBel, A generalized a-Wright convexity and related function equation, Ann. Math. Silesianae, 10 (1996), 7-12.

[4] Zs. PÁLeS, On two variable functional inequality, C. R. Math. Rep. Acad. Sci. Canada, 10 (1988), $25-28$. 